encephalopathy. The 2-month-old infant in the Bangkok study had a low birth weight and hepato-splenomegaly, while the 2-year-old was reported to have a normal development up to the onset of stroke.

\title{
SSPE AND NEONATAL MEASLES
}

A case of subacute sclerosing encephalitis in a child, aged 3 years 6 months, who had contracted measles from his mother on day 27 as a neonate, is reported from Akita University School of Medicine, Japan. He presented with acute cerebellar ataxia which gradually improved, but mental deterioration and head nodding developed. The EEG showed diffuse slowing and periodic discharges that synchronized with the nodding. Measles antibody titers in the CSF and serum were abnormally elevated. MRI showed diffuse high intensity T2-weighted images in the white matter. Treatment with oral isoprinosine and intraventricular interferon failed to stem the relentless progression to a bedridden, mute stage within 3 months. This was followed by a plateau and later, a gradual but limited clinical improvement and MRI evidence of progressive cerebral atrophy. (Sawaishi Y, Abe T, Yano T, Ishikawa K, Takada G. SSPE following neonatal measles infection. Pediatr Neurol Jan 1999;20:63-65). (Respond: Dr Yukio Sawaishi, Department of Pediatrics, Akita University School of Medicine, Hondo 1-1-1, Akita 010-8543, Japan).

COMMENT. Measles virus infection under 1 year of age is a risk factor of SSPE Immaturity of the brain at the time of measles infection may predispose to the SSPE.

\section{ATTENTION AND COGNITIVE DISORDERS}

\section{ADHD AND COMORBID COORDINATION DISORDER}

The cooccurrence of attention deficits and motor incoordination or clumsiness was examined at the University of Goteborg in a population study of 400 seven-year-old children attending mainstream schools in Karlstad, Sweden. ADHD, developmental coordination disorder (DCD), and combined deficits in attention, motor control, and perception (DAMP) were found in 6.1\%. Severe ADHD alone occurred in 2\% and moderate ADHD in 5.4\%. The boy:girl ratio was 2:1 for severe DAMP and 6.2:1 for severe ADHD alone. Considerable overlap of attention deficits and motor clumsiness was present. One half the children with ADHD had moderate motor incoordination (DCD), and one in 5 was severely affected. Similarly, children with DCD frequently met criteria for the diagnosis of ADHD. Findings were unchanged at follow-up examinations 8 months later. 'Soft signs' were highly reproducible, with excellent agreement between neurologic exams and observations of motor dysfunction reported by physical education teachers. Parent reports of ADHD were confirmed by teacher observations in more than $90 \%$ of cases, whereas $40 \%$ of teacher diagnoses of ADD were not reported by parents in the home setting. DSM-III-R criteria for ADHD tended to exclude many ADD children with a high degree of classroom dysfunction. Those with subtype ADD or DAMP had higher classroom-dysfunction scores than those with mainly hyperactivity or ADHD. DAMP is a valid diagnostic subtype of ADHD. (Kadesjo B, Gillberg C. Attention deficits and clumsiness in Swedish 7-year-old children. Dev Med Child Neurol Dec 1998;40:796-804). (Respond: Christopher Gillberg MD PhD, Department of Child and Adolescent Psychiatry University of Goteborg, Sahlgren University Hospital, S-41345, Goteborg, Sweden).

COMMENT. A neurologic examination to uncover soft signs, motor 
incoordination, and sensori-perception impairments ("minimal brain dysfunction" or MBD) is an important part of the work-up of a child at risk of ADD. The omission of signs of impaired motor control and perception from the diagnostic criteria for ADHD excludes many ADD children with significant classroom dysfunction. A return to the former, more objective MBD criteria, in addition to the subjective, symptomatic criteria for ADHD, would lead to the earlier recognition of children with increased risk of classroom dysfunction sufficient to demand medical, psychological, and educational therapy. See Progress in Pediatric Neurology III, PNB Publ, 1997;pp204-205, for a review of previous Scandinavian reports of the DAMP syndrome and ADHD.

Huttenlocher PR (1990) and Millichap JG (1974) have emphasized the value of neurologic soft signs in the prediction of learning disabilities in preschool children and the correlation with hyperactive behavior and response to methylphenidate. (see Progress in Pediatric Neurology I, 1991;pp167-8).

\section{ATTENTION DEFICITS, ADHD, AND COMPLEX PARTIAL SEIZURES}

The degree of attention deficits in children with complex partial seizures (CPS), with and without ADHD, were compared with that found in children with ADHD but without epilepsy, in a study at the Department of Educational Psychology, University of Texas, Austin. A computerized performance test (CPT), evaluating sustained attention, inhibition of response, response time, and consistency of response, was completed by 12 children with CPS and ADHD, 21 with CPS alone, 22 with ADHD alone, and 15 controls. CPT performance was unrelated to IQ scores on the WISC-R. Children with CPS and ADHD had the lowest scores on the CPT. Children with CPS had impaired sustained attention regardless of the diagnosis of ADHD. Antiepileptic medication, usually carbamazepine, taken by $78 \%$ of the patients with CPS was considered an unlikely cause of the attention deficits. Methylphenidate administered to patients with ADHD improved performance of the CPT in both the group with seizures and without. (Semrud-Clikeman M, Wical B. Components of attention in children with complex partial seizures with and without ADHD. Enilepsia February 1999;40:211-215). (Reprints: Dr M Semrud-Clikeman, Department of Educational Psychology, SZB 504, University of Texas, Austin, TX 78413).

COMMENT. Complex partial epilepsy is associated with attention problems that interfere with learning and memory. In patients with CPE complicated by $\mathrm{ADHD}$, methylphenidate has a similar beneficial effect on attention as that observed in children with ADHD without seizures.

An EEG is indicated in children with ADHD who have episodes of confusion or staring that interfere with attention and learning. Treatment with carbamazepine may be advisable before the initiation and addition of stimulant medication. CBZ-induced lowering of MPH blood levels is reported (see p.15).

\section{ARACHNOID CYST DECOMPRESSION AND COGNITIVE RECOVERY}

A 20-year-old right-handed male with a large left temporal arachnoid cyst, discovered incidentally by CT following a kick to the head with brief loss of consciousness, is reported from the University of Texas Medical Branch, Galvestan, TX. MRI revealed a mass effect with midline shift and left temporal lobe hypoplasia. Severe headaches had increased in frequency for one year after the head trauma. Early history and developmental milestones were normal, but learning disabilities and behavior disorder lead to school drop-out at 11 th grade. Pre-surgery psychological testing revealed a borderline verbal IQ of 76 and average performance IQ of 90 , with clinical discrepancy in verbal (left hemisphere) and visual-perceptual/constructional (right hemisphere) skills. 